\title{
Korrespondenzen.
}

\section{Bemerkungen zu dem Aufsatz von Haubold') „Der soziale Kampf gegen die malignen Geschwulste in Frankreich und seine Lehren".}

Von Dr. Siegfried Wolff in Eisenach.

Ha ubolds Aufsatz und die Bemerkungen der Schriftleitung sind sehr interessant. Der fernerstehende Praktiker findet aber, daß es eigentlich genügend Ausschüsse, Komitees, Fürsorgestellen, Fürsorgevereinigungen gibt. Dem therapietreibenden Praktiker fehlt in alledem nur eines, nämlich das Wichtigste: eine Stelle, diebei rechtzeitiggestellter Diagnosesofortauch da Hilfebringt, wo kein Geldzur Verfügung steht.

Die ganze Fürsorge ist wertlos, wenn sie in wirklich schweren Fällen versagt. Daß das tatsächlich in einer großen Anzahl von Fällen, gerade Fällen, in denen sofortige, kostspielige oder langdauernde Hilfe nottut, so ist, und zwar weil infolge bureaukratischer Einstellung der ganzen. Fürsorgen nie eine Instanz da ist, die sofort entscheiden kann, soll ausführlich a. a. O. mit Beweisen dargelegt werden.

Hier ein Beweis dafür, daß es gar nichts nützt, rechtzeitig die Krebsdiagnose zu stellen:

Bei einem $3 / 4$ Jahre alten Kinde wird bei der 1. Untersuchung die Diagnose auf Nebennierensarkom gestellt. Bei der Operation, die die Diagnose bestätigt, wird der Tumor, der kindskopigroß ist, entfernt, und das Kind übersteht die Operation und eine schwere Pneumonie überraschend gut. Vier Wochen nach der Entlassung aus dem Krankenhaus zeigt sich ein walnußgroßer Tumor in der Operationsnarbe, der exzidiert wird. Nach 2 Monaten kommt das Kind wieder mit mehreren Tumoren im Abdomen. Es wird nach Berlin geschickt, mit Radium bestrahlt und als es etwa 4 Wochen nach der Bestrahlung sich wieder vorstellt, sieht es blühend aus, die Tumoren sind restlos verschwunden, es hat sehr zugenommen, Zähne bekoinmen und laufen gelernt. Nach etwa weiteren 3 Monaten zeigen sich erneut Tumoren im Abdomen, die in gleicher Weise beseitigt werden.

Als sich dann nach 6 Monaten erneut Tumoren zeigten, waren die Eltern, die, kleine Geschäftsleute, bisher alles bezahlt hatten, nicht mehr imstande, die Radiumbestrahlungen, die unverhältnismäßig teuer sind, zu bezahlen und das Kind ging elendiglich zugrunde.

$\mathrm{Ob}$ es zu retten gewesen wäre, ob nicht doch einmal trotz Radium der Tumor gesiegt hätte, ist eine andere Frage. Schon aus wissenschaftlichen Gründen hätten weitere Bestrahlungen unentgeltlich möglich sein müssen. Trotz aller Bemühungen war aber unentgeltliche Hilfe nicht zu haben. Was nützt also alle Aufklärung und Frühdiagnose? Was nützen alle Krebsinstitute und Forschungsheime!

1) D. m. W. 1930 Nr. 46. 
Was nottut ist eine Stelle, die sofort-dennes kommt ja auf Tage an - Hilfe bringt, gleichgültig, ob sie bezahlt werden kan oder nicht. Die Erforschung, ob und wievielund von wem die Hilfebezahltwerden kann, kann ruhiginbeliebtbureaukratischer Langsamkeit vorsichgehen. Dies nenne ich sozialen Kampigegen die malignen Gesehwülste. 